\title{
Capturing Dynamics of Pulsed Laser Induced Melting and Rapid Solidification in Aluminum Polycrystals with Nanoscale Temporal Resolution In-situ TEM
}

\author{
Jörg M.K. Wiezorek ${ }^{1}$, Joseph T. McKeown ${ }^{2}$, Kai Zweiacker ${ }^{1}$, Thomas LaGrange ${ }^{2}$, Bryan W. Reed ${ }^{2}$, Can \\ $\mathrm{Liu}^{1}$ and Geoffrey H. Campbell ${ }^{2}$ \\ 1. Department of Mechanical Engineering and Materials Science, University of Pittsburgh,Pittsburgh, \\ PA 15261 \\ 2. Condensed Matter and Materials Division, Lawrence Livermore National Laboratory, Livermore, \\ CA 94556
}

The unprecedented spatiotemporal resolution offered by the dynamic transmission electron microscope (DTEM) facilitates unique studies of irreversible transitions in condensed matter [1]. In prior work we utilized the DTEM for single-shot nano-second temporal resolution bright field imaging and diffraction studies of rapid solidification (RS) in $\mathrm{Al}, \mathrm{Cu}, \mathrm{Ag}$ and $\mathrm{Al}-\mathrm{Cu}$ alloy thin films [2-5]. Recent upgrades to the DTEM instrument enable single-pump/multiple-image-acquisition experiments (image series), which hold prospect to greatly reduce quantitative uncertainties in measurements, e.g. transformation front velocity, during RS, and increase by about one order of magnitude the data density obtained from direct observations of the dynamics of the irreversible transformation processes [6]. Here we report and discuss first results of in-situ TEM investigations of the dynamics of pulsed-laser induced melting and $\mathrm{RS}$ in Al thin films performed with the upgraded DTEM. Thin Al films, 80nm-160nm thick, were deposited by e-beam evaporation on square windowed TEM grids with $50 \mathrm{~nm}$ thick amorphous $\mathrm{Si}_{3} \mathrm{~N}_{4}$ membranes and melted with a single $15 \mathrm{~ns}, 1064 \mathrm{~nm}$ laser pulse, defining time $\mathrm{t}_{0}$ (Fig. 1). Image series comprised of nine frames have been acquired with preselected $20 \mathrm{~ns}$ exposures per frame and $0.2 \mu$ s time steps between frames and also for 50ns exposures and inter-frame time steps of $2 \mu \mathrm{s}$. The resulting image series spanning at least $\sim 1.8 \mu$ s (e.g. Fig. 1) and up to $\sim 20.4 \mu$ s have been acquired for systematically varied pre-delay times, $\Delta \mathrm{t}$, in the range of $-0.2 \mu \mathrm{s}$ to $40 \mu \mathrm{s}$. Dynamics of the morphological and structural changes in the nano-crystalline Al thin films and the kinetics during the pulsed laser induced sequences of melting and subsequent RS have been determined from direct observations. Bright field imaging showed (e.g. Fig. 2) that the laser pulse induced irreversible transformation involves the preferential melting along grain boundaries and development of a 'mushy' two-phase region during the melting sequence. Subsequently solidification initiates with a transformation interface that is rough on the scale of the adjacent crystalline grains. Driven by the thermal gradient the solidification interface transitions to a planar morphology, which supports growth of columnar crystals to the center of the melt pool. Effects from the Si frame support of the TEM transparent square windows, which represents a heat sink, on the solidification velocity measurements in the DTEM have been studied systematically. Kinetic details of the Al thin film melting and the solidification sequences under the driven conditions are analyzed quantitatively and discussed. Post-mortem TEM analysis have been performed to evaluate crystallographic details of the directional solidified Al grains relative to the original film.

\section{References}

[1] King, W.E., et al., Journal of Applied Physics (2005) 97: 111101.

[2] Kulovits, A.K., et al., Microscopy and Microanalysis (2011) Vol. 17(Suppl. 2): 506.

[3] Kulovits A, et al., Philosophical Magazine Letters (2011) 91: 287.

[4] McKeown, J.T., et al., Microscopy and Microanalysis (2012) Vol. 18(Suppl. 2): 602.

[5] McKeown, J.T., et al., Acta Materialia (2014) 65: 56. 
[6] LaGrange T., et al., Micron (2012) 43: 1108.

[7] Work was performed under the auspices of the U.S. Department of Energy by the Lawrence Livermore National Laboratory and supported by the Office of Science, Office of Basic Energy Sciences, Division of Materials Sciences and Engineering, of the U.S. Department of Energy under contract No. DE-AC52-07NA27344. The research activities at the University of Pittsburgh received support from the National Science Foundation, Division of Materials Research, Metals \& Metallic Nanostructures program through Grant No. DMR 1105757.

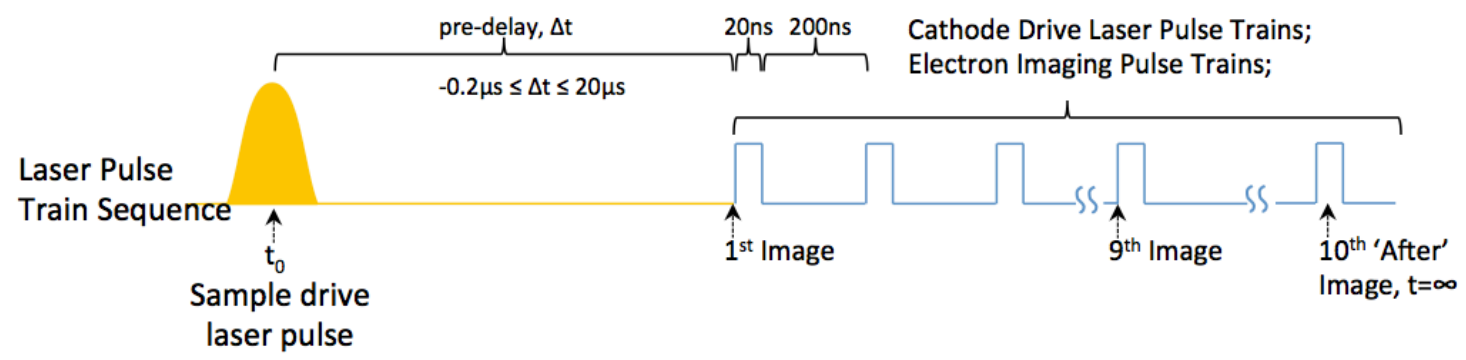

FIG. 1. Schematic of an example experimental sequence of sample melt laser pulse, delay time and the cathode drive laser pulse train for the nine-frame bright field TEM image series.

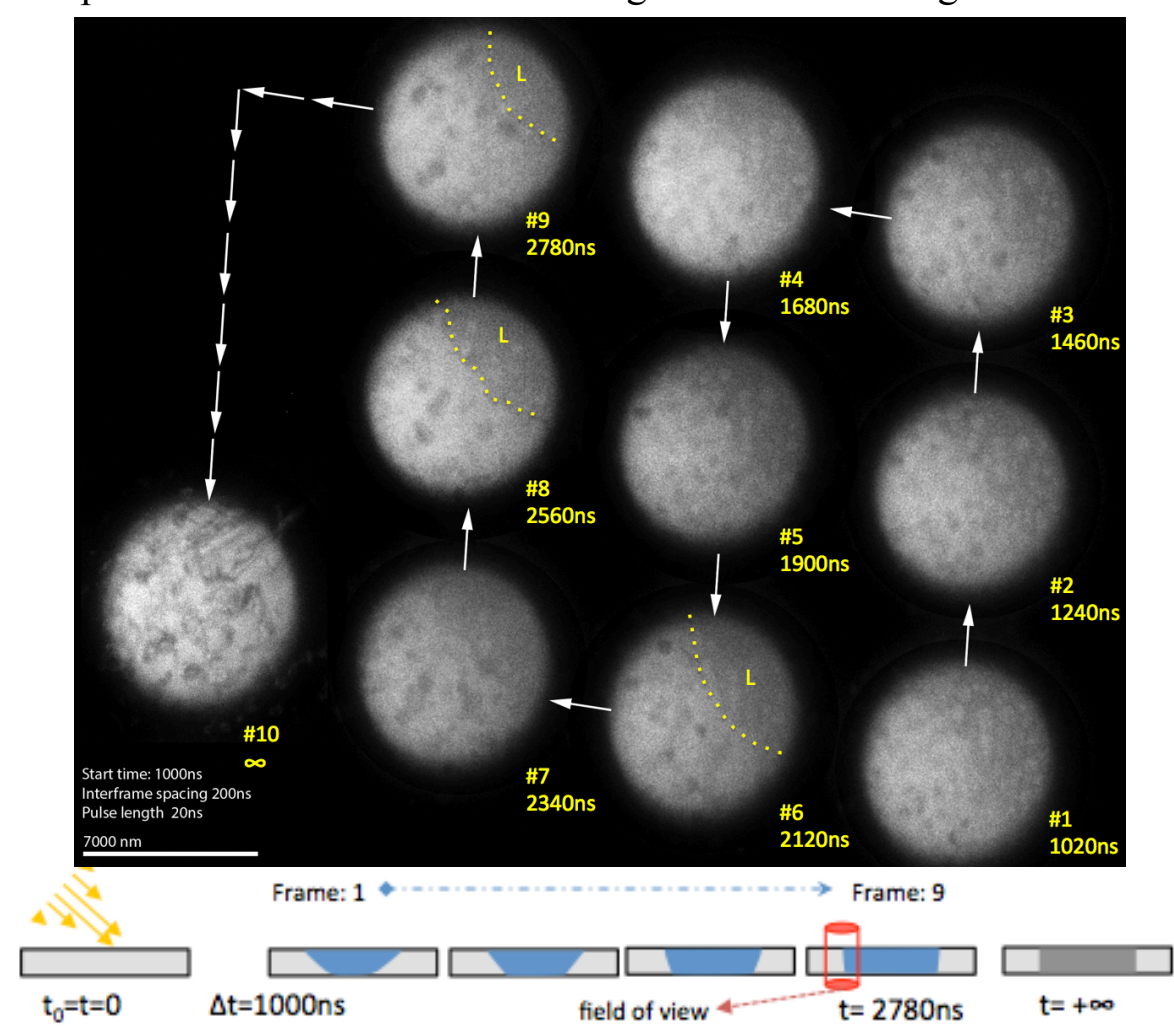

FIG. 2. Image-series (\#1-\#9) and post-solidification (\#10) state for $160 \mathrm{~nm}$ Al film, capturing dynamics of the morphological evolution during melting and onset of rapid solidification in $220 \mathrm{~ns}$ time-steps, for pre-delay $\Delta \mathrm{t}=\mathrm{t}-\mathrm{t}_{0}=1000 \mathrm{~ns}(\# 1)$. The $7 \mu \mathrm{m}$ diameter field of view is located at the edge of the $\sim 35-40 \mu \mathrm{m}$ diameter melt pool, as indicated graphically in the schematic (at bottom, not to scale), where blue (darker shade) indicates liquid Al, and gray (lighter) shade solid Al. Dashed lines mark approximate locations of the liquid-solid interface, with $\mathrm{L}=$ liquid, in frames \#6, \#8 and \#9. 